Original Article (short paper)

\title{
Blood glucose threshold determination is stage length dependent
}

\author{
Gilmar de Jesus Esteves ${ }^{1,2,3}$ (D), Paulo Eduardo Pereira ${ }^{1,2,4,5}$ (D), Yuri Lopes Motoyama ${ }^{1,6}$ (D), \\ Mariam Mian Bouchnak ${ }^{1}$ (D), Paulo Henrique Silva Marques de Azevedo ${ }^{1,4}$ \\ ${ }^{1}$ Grupo de Estudos e Pesquisas em Fisiologia do Exercício, Santos, Universidade Federal de São \\ Paulo, São Paulo, SP, Brazil. \\ ${ }^{2}$ Faculdade Praia Grande, Praia Grande, SP, Brazil. \\ ${ }^{3}$ Faculdade de São Vicente, São Vicente, SP, Brazil. \\ ${ }^{4}$ Programa de Pós-graduação em Ciências do Movimento Humano e Reabilitação, Universidade \\ Federal de São Paulo, Santos, SP, Brazil. \\ ${ }^{5}$ Universidade Metropolitana de Santos, Santos, SP, Brazil. \\ ${ }^{6}$ Universidade Paulista, Santos, SP, Brazil.
}

\begin{abstract}
Aim: This study aimed to verify whether different stage length affects the intensity of the Blood Glucose Threshold (BGT), and the agreement between evaluators for BGT determination. Methods: Fourteen subjects attended the laboratory during the first session to perform anthropometric measures and become familiar with procedures. In the following three sessions, subjects performed an incremental test on the ergometer bicycle and in each test a different protocol was performed in randomized order (1,3- and 5-min stage) to identify BGT. Three different evaluators determined the BGT. Results: Our data show that the BGT is stage length-dependent $(1,3-$ and 5 -min; $\mathrm{P}<0.0001)$. The intraclass correlation coefficient showed that there was a strong correlation among evaluators for all protocols (ICC $=0.8$ to $1 \mathrm{~min}$; ICC $=0.8$ to $3 \mathrm{~min}$; and ICC 0.9 to $5 \mathrm{~min}$ ). However, one evaluator determined the BGT at a higher intensity than others. The peak load was lower at long stage length. Conclusion: We concluded that stage length influences the BGT intensity determination. The BGT presents a good agreement among evaluators. However, a minimum of two evaluators is needed for BGT determination. The peak load is affected by stage length.
\end{abstract}

Keywords: aerobic fitness, testing, metabolism, performance.

\section{Introduction}

We can evaluate aerobic capacity through different methodologies ${ }^{1}$ and physiological variables (e.g., lactate, glucose, ventilatory responses; and heart rate variability) that change in response to incremental exercise intensity ${ }^{1-3}$. Currently, the gold standard for aerobic capacity analysis is the Maximal Lactate Steady State (MLSS) ${ }^{4}$. However, the MLSS assessment needs the subject's engagement in 3 to 6 days of tests with a 30-min duration each one, which has low practical appeal for athletes and subjects looking for better health. The previous studies have already verified that the called Blood Glucose Threshold (BGT) can estimate the $\mathrm{MLSS}^{5-7}$. Sotero, Pardono, Landwehr, Campbell, Simoes ${ }^{5}$ compared the BGT with the MLSS using an electroenzymatic method to determine the blood glucose and lactate concentration, a device with high precision. However, this equipment is expensive and mainly used in a laboratory for research propose. On the other hand, Motoyama et al. ${ }^{6}$ used a portable glucose meter, a cheaper device to analyze the BGT, but with lower precision.

The BGT is the intensity corresponding to the lowest blood glucose concentration during an incremental exer- cise test ${ }^{1,8}$. Currently, BGT is easy to apply and inexpensive if a portable glucose meter is used. It has also been used to prescribe exercise intensity in endurance training ${ }^{9}$. Moreover, BGT is a change-sensitive method after a longterm endurance-training program ${ }^{8,9}$. When the BGT is compared to MLSS the bias showed were $0.01 \pm 0.92 \mathrm{~km}$. $\mathrm{h}^{-1}$ using an electroenzymatic method and $0.21 \mathrm{~km} \cdot \mathrm{h}^{-1}$ (confidence interval from -1.26 to 1.29) using a portable glucose meter. Therefore, both devices seem to be useful for BGT determination. One study compared the BGT with MLSS in a stage length of $3 \min ^{6}$ and another one with variable duration (800-meter run with increased intensity at each stage $)^{5}$.

Despite the ease of the BGT determination (U-shape glucose response) ${ }^{1}$, we do not know if the stage duration influences the BGT intensity determination, like other physiological variables (e.g., lactate, ventilatory thresholds $)^{10}$. Also, a previous study indicates that 3-min stage would represent a reliable metabolic index when it was assessed metabolic variables (e.g. lactate, $\mathrm{CO}_{2}$ output, $\mathrm{O}_{2}$ uptake) in an incremental test ${ }^{10,11}$ Recent data show that lower stages length can overestimate the BGT intensity 
while longer stages length can underestimate metabolic variables during an incremental test ${ }^{12}$. Another point that needs to be clarified is the objectivity of the BGT protocol. Because of the easy BGT determination, we were expecting that this parameter would represent the high agreement among evaluators. High objectivity would be suitable for studies comparison, coach assessments of athletes and general population.

Thus, this study aimed to verify whether different stage length influences the intensity associated with the BGT. Moreover, we checked whether BGT has good objectivity among different evaluators.

\section{Methods}

\section{Participants}

We recruited, by convenience, fourteen physically active men. This number was based on a previous pilot study calculated using $\alpha=5 \%$ and $\beta=90 \%$. Volunteers had the following characteristics: height $1.74 \pm 0.07$; weight $75.16 \pm 10.48$; and age $23.75 \pm 2.97$, with recreational cycling experience for at least six months. We chose recreational cyclists to have more homogeneity and because they were physical education students in our university. We informed the volunteers about the procedures, possible discomforts, and risks involved in this study. They could not present any disease (diabetes, cardiomyopathy, etc.) or muscle injury that could interfere with the outcome of this study. We warned the volunteers not to consume any ergogenic resources or supplements during all intervention period and not to include any additional training during the data collection. A local research ethics committee approved the experimental procedures of this study (number 283.285/2014), and this work is complying with the ethical guidelines of the Helsinki Declaration ${ }^{13}$. We declare there is no conflict of interest.

\section{Experimental design}

All subjects attended the laboratory on four occasions separated by 72 to 120 hours. In the first session, subjects performed anthropometric measures and became familiar with procedures. In the following three sessions, we conducted the tests in crossover and randomized design. Subjects performed three incremental tests on an ergometer bicycle with a mechanical braking system (Monark $^{\circledR}$, model 828E). In each test, a different protocol was performed (1,3- and 5-min stage) to identify BGT.

We conducted the tests at the same time of the day (morning) to reduce the physiological variability related to the circadian rhythm. The laboratory temperature was kept at $21{ }^{\circ} \mathrm{C}$. We instructed the subjects to maintain a similar diet $24 \mathrm{~h}$ before an experiment session and to refrain from eating $3 \mathrm{~h}$ before the experiment. The same researcher encouraged the subjects verbally to keep the highest rhythm during all tests.

\section{Procedures}

Maximal incremental test

We submitted the subjects to three maximal incremental ergometer bicycle tests on different days. The trials were randomized but not counterbalanced because of the number of subjects. After $3 \mathrm{~min}$ of warm-up, with a load of 50 Watts, the subjects started the incremental test. We incremented 50 Watts each 1, 3 or 5 min stage (in different sessions $)^{10}$. The subjects kept cadence at 60 RPM. The tests ended when subjects reached the voluntary exhaustion (subject report) or the inability to maintain the exercise cadence. The participants were verbally encouraged to do the maximal performance they could do during the exercise.

It was taken a $5-\mu \mathrm{L}$ blood sample from the earlobe during the last fifteen seconds of each stage (1, 3- or 5min). The sample was immediately used for the blood glucose analysis by a portable digital blood glucose meter (Bayer ${ }^{\circledR} /$ Breeze ${ }^{\circledR} 2$ model). Before each blood collection, asepsis in the site was performed to avoid contamination by sweating or other material. Blood glucose was expressed in $\mathrm{mg} / \mathrm{dL}$.

HR parameters were measured continuously during the maximal incremental test through the heart rate monitor Polar ${ }^{\circledR}$ RS800. The HR was measured during the last fifteen seconds of each stage (1, 3- or 5-min).

Blood glucose threshold determination

Three independent and experienced evaluators determined the BGT by visual inspection of the glucose curve (U-shaped). The BGT was determined to be the exercise intensity at which the lowest blood glucose value occurred, followed by one increase during the incremental test.

\section{Statistical analysis}

Values are presented as mean and standard deviation. The Shapiro-Wilk and Levene's tests were performed to verify the normality and homogeneity of the data, respectively. For practical application purposes, we showed raw data but, to solve some problems with non-normal data, we used a natural logarithm to compare means through $3 \mathrm{x}$ 3 three-way ANOVA with repeated measures (stages length $\mathrm{x}$ evaluators). We analyzed the eta-partial squared to determine the effect size. We applied the intraclass correlation coefficient (ICC) to evaluate within and betweenrater objectivity. To verify statistical significance, in all comparisons, we considered confidence intervals of $95 \%$ and $\mathrm{P} \leq 0.05$.

To check the qualitative outcomes and the probability to replicate the same results (i.e., the magnitude of 
the evidence), we applied the Bayes Factor hypothesis testing analyses. The posterior odds have been corrected for multiple testing by fixing to 0.5 the prior probability that the null hypothesis holds across all comparisons ${ }^{14}$. Individual comparisons are based on the default t-test with a Cauchy $(0, r=1 /$ sqrt(2)) prior. The "U" in the Bayes factor denotes that it is uncorrected ${ }^{14-16}$. The outcomes were classified as anecdotal (BF10 $=1$ to 3 ), moderate ( 3 to 10$)$, strong (10 to 30 ), very strong (30 to 100 ) and extreme $(>100)$ favoring the alternative hypothesis; or anecdotal (BF10 $=1$ to 0.33$)$, moderate $(0.33$ to 0.1$)$, strong $(0.1$ to $0.03)$, very strong $(0.03$ to 0.01$)$ and extreme $(<0.01)$ favoring the null hypothesis (Lee and Wagenmakers' classification $)^{15-17}$. To calculate the probability to find the same results again, we divided the actual BF10 value by $\mathrm{BF} 10+1$. We made all $\mathrm{BF}$ analysis through the $\mathrm{JAMOVI}^{\circledR}$.

\section{Results}

\section{Submaximal parameters}

There was a difference for BGT intensity among different stage length $\left(\mathrm{P}<0.0001 ; \mathrm{\eta}_{\mathrm{p}}^{2}=0.37\right)$ (Figure 1). The 1-min stage length was different from $3(\mathrm{P}=0.045 ; \mathrm{BF}=$ $130.2)$ and 5 -min $(\mathrm{P}<0.001 ; \mathrm{BF}=150.4)$ ones. Although the null-hypothesis statistical testing was not significantly different between 3 vs $5 \min (\mathrm{P}=0.102)$, the Bayes Factor showed a chance of $99 \%$ of BGT intensity for 3-min length to be higher than 5 -min $(B F 10=100)$. It shows that BGT is length stage-dependent.

The heart rate corresponding to the BGT was not different for stage length between 1 vs $3 \min (\mathrm{P}=1.0, \mathrm{BF} 10=$ $0.309)$ and 1 vs $5 \min (\mathrm{P}=0.39, \mathrm{BF} 10=2.904)$ (Figure 2$)$. Although the null-hypothesis statistical testing was not significantly different for 3 vs 5 min $(P=0.16)$, the Bayes Factor showed a chance of $97 \%$ of HR for 3-min length to be higher than $5-\min (\mathrm{BF} 10=29.436)$ one. Therefore, caution is needed for training prescription using HR to monitor training intensity.

The intraclass correlation coefficient (ICC) showed that there was a strong correlation (agreement) between the evaluators for all protocols (Figure 3). However, we found difference from evaluator 3 to evaluator $1(\mathrm{P}=0.02$; $\mathrm{BF}=1.638)$ and $2(\mathrm{P}=0.001 ; \mathrm{BF}=12.693)$. The evaluator 3 determined the BGT at higher intensities than evaluators 1 and 2 ( $62 \%$ and $93 \%$ of the chance to be different, respectively).

\section{Maximum parameters}

The time to exhaustion (test duration) was different among stage length $\left(\mathrm{P}<0.001 ; \mathrm{\eta}_{\mathrm{p}}^{2}=0.97\right)$. The 5 -min stage was the longer protocol $(28.93 \pm 3.50 \mathrm{~min})$; 1 -min was the shorter $(8.29 \pm 0.99 \mathrm{~min})$ and 3-min protocol falling in between $(19.71 \pm 2.55 \mathrm{~min})$. According to Bayes
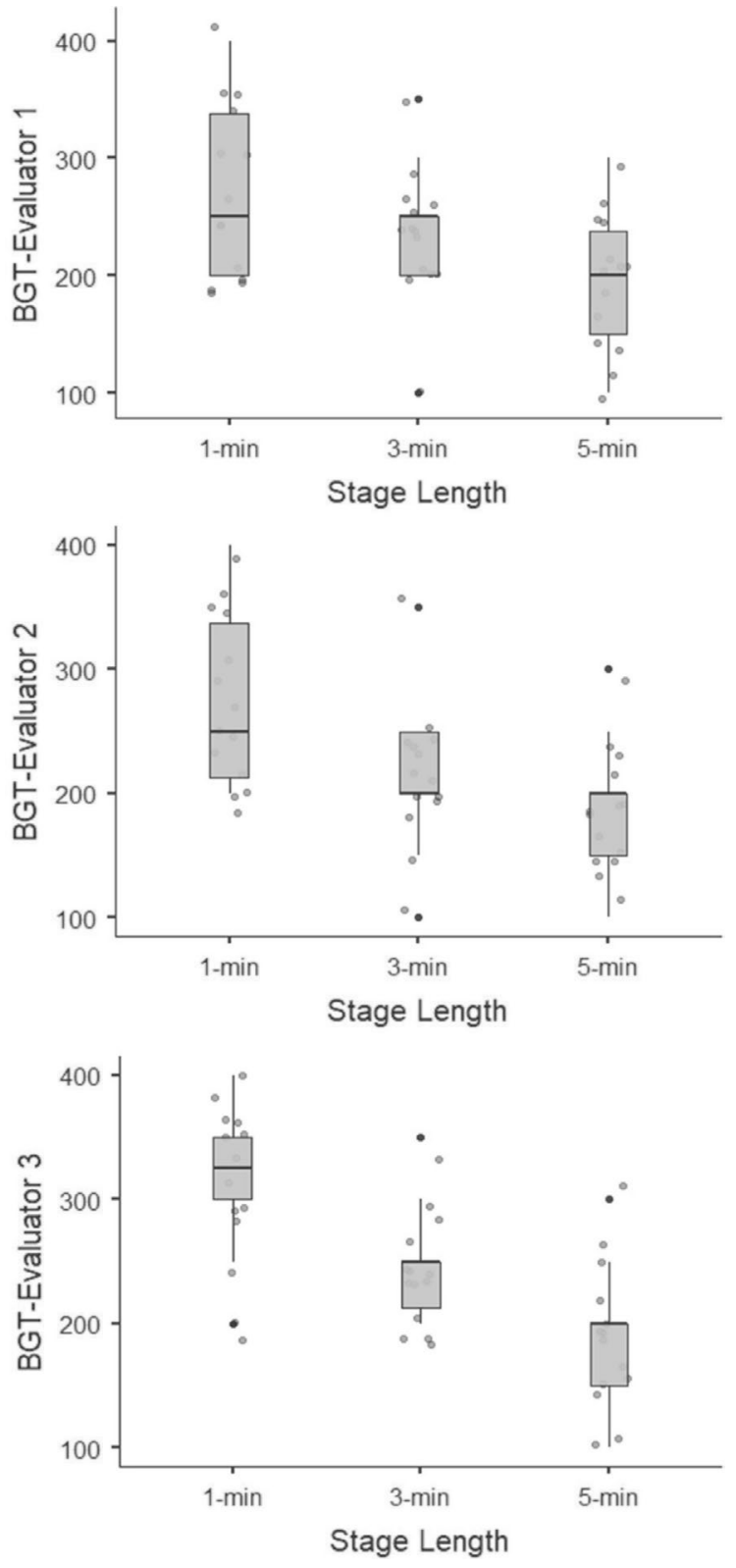

Figure 1 - BGT Intensity (mean) for each evaluator in each stage length.

Factor analyzes there is a chance of $99 \%$ to observe a difference among all stage lengths for time to exhaustion.

The maximum power obtained was different among stage length $\left(\mathrm{P}<0.001 ; \eta_{\mathrm{p}}^{2}=0.88\right)$. All comparisons between stage length had a $\mathrm{P}<0.001$. The Bayes Factor analyses show that the 1-min stage length has a probability of $96 \%(\mathrm{BF} 10=22.504)$ and $99 \%(\mathrm{BF} 10=761.448)$ to be higher than 3 and $5 \mathrm{~min}$, respectively. The 3-min stage length has $99 \%$ of probability to be higher than 5 -min $(\mathrm{BF} 10=149)$ one. The 5 -min stage had the lower load achieved (289 \pm 35 Watts); 1-min had the higher load (414 \pm 49.7 Watts); and 3-min protocol falling in between (329 \pm 42.6 Watts). 


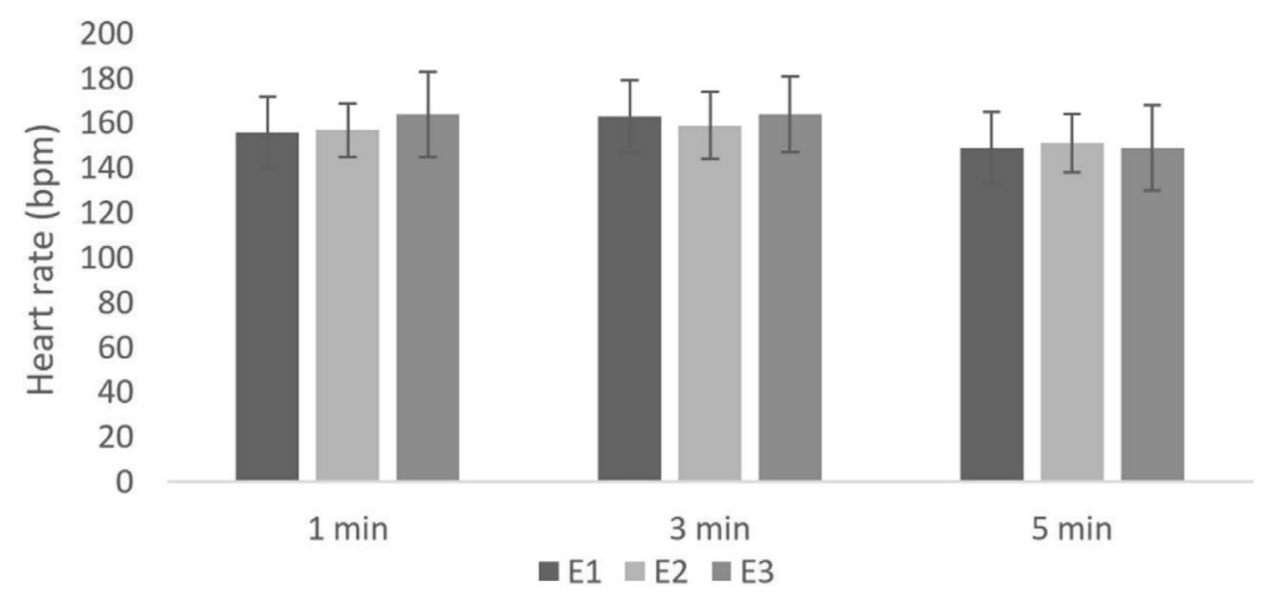

Figure 2 - The heart rate corresponding to the Blood Glucose Threshold for each Evaluator in E1 = Evaluator 1, E2 = Evaluator 2, E3 = Evaluator 3.

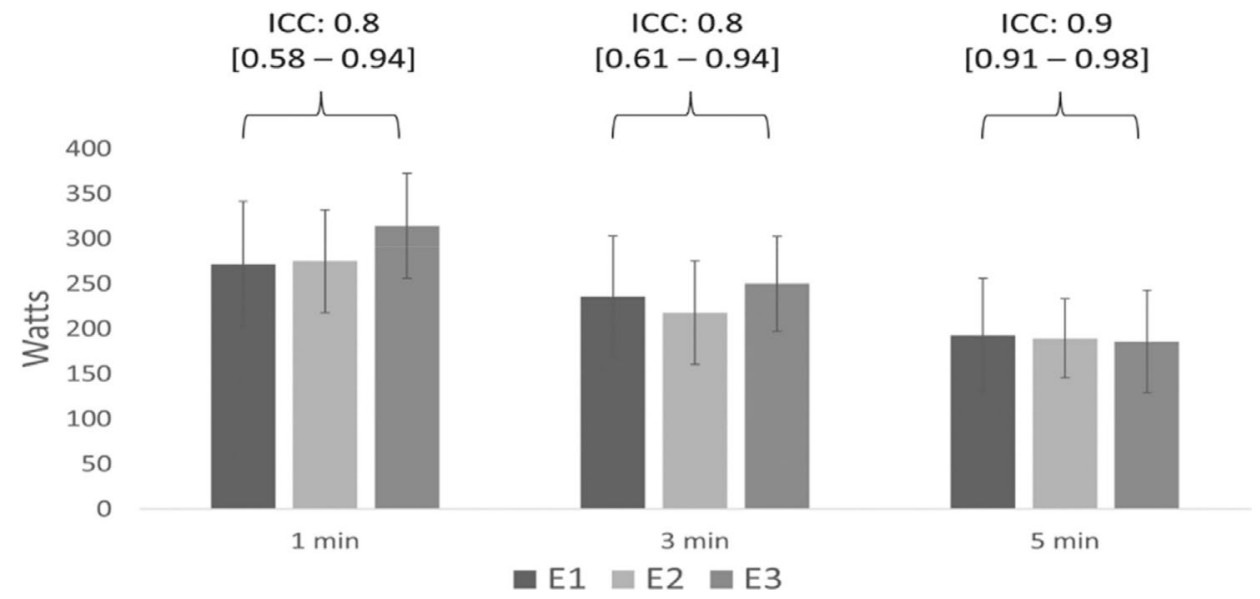

Figure 3 - Intraclass correlations among evaluators for each stage length. E1 = Evaluator 1, E2 = Evaluator 2, E3 = Evaluator 3. ICC = Intraclass Correlation Coefficient. $P>0.05$ among all evaluators.

The maximum heart rate was not different among stage length $\left(\mathrm{P}=0.24 ; \eta_{\mathrm{p}}^{2}=0.11\right)$. The values obtained were $183 \pm 8.91$ (1-min); $187 \pm 11.15$ ( $3 \mathrm{~min})$; and $182 \pm$ 10.79 ( $5 \mathrm{~min})$. The Bayes Factor showed a chance of $56 \%$ of similarity for maximum heart rate among stage length.

\section{Discussion}

The present study is the first one to verify whether different stage length influences the intensity associated with the BGT and the objectivity of BGT determined by different evaluators. Our main findings are that the stage length in an incremental test influences the BGT intensity. Also, there is a high intraclass correlation (agreement) among different evaluators for BGT intensity determination, regardless of stage length. However, one evaluator determined the BGT at higher intensities. Furthermore, the maximum intensities and time to exhaustion were modulated by stage length.

A previous study published by our group has postulated that the MLSS can be estimated by BGT using the protocol with a 3-min stage length in an incremental exercise $^{6}$. Starting from this point makes sense to use 3-min one to increase workload (stage length) in an incremental test to provide reliable and valid measures of both glucose and lactate values ${ }^{7,10}$. Here our data shows that there is a difference for BGT intensity when different stage length $(1,3$ and $5 \mathrm{~min})$ are applied, even for different evaluators (Figure 1). At the beginning of the incremental test, with the exercise intensity increments, blood glucose concentration decreases mainly due to its uptake by skeletal muscle. At one point, after glycaemia reaches a minimum (BGT determination point), there is an exponential increase in glucose indexes mediated mainly by the rise in the release of catecholamines, glucagon, cortisol, and $\mathrm{GH}^{1,18}$, which gives the U-shape form ${ }^{1}$. An explanation for the difference between stages in our study would be due to the difficulty of metabolism in stabilizing these physiological responses, overestimating the value of the BGT in 1-min stage length, which could be the same for $3 \mathrm{~min}$, and/or underestimating in the 5-min stage length. 
Since the BGT intensity is slightly higher than MLSS when applied a stage of $3 \mathrm{~min}^{5}$, or fixed lap distance $(800 \mathrm{~m})^{6}$, a further study must compare the BGT and MLSS with longer stage length to improve precision. To know the best stage length in the incremental aerobic test is essential to make precise evaluations to prescribe exercise intensity. Spending little time to identify metabolic thresholds is useful to minimize the discomfort of the subject.

Usually, two or more experienced evaluators are needed to identify the intensity at which metabolic threshold occurs by other physiological parameters ${ }^{19}$. Despite the ease in determining the BGT, it is necessary to interpret the glycemic values by different experienced evaluators, increasing the objectivity. In this study, we showed that there was a difference from evaluator 3 to evaluator 1 and 2, although we verified a strong intraclass correlation among evaluators (Figure 3). The high ICC was tested in the three analyzed stages length. We hypothesized that although the stage length could affect the BGT intensity, it is still possible to identify a minimum point in blood glucose. However, evaluator 3 determined the BGT at higher power. Therefore, a minimum of 2 evaluators is needed to improve the BGT objectivity.

The heart rate at BGT was not different between stages length according to null-hypothesis significant test. It can have a practical significance because regardless of the intensity associated with the lower blood glucose concentration, we could prescribe the training sessions based on heart rate. However, the Bayes Factor showed a significant chance $(97 \%)$ of the heart rate in 3-min stage length to be higher than with $5 \mathrm{~min}$. Therefore, the person could do a training session in an inadequate intensity. Caution is needed when using HR to prescribe the training intensity.

The time to exhaustion (test duration) was different among stage length $\left(\mathrm{P}<0.001 ; \eta_{\mathrm{p}}^{2}=0.97\right)$. The 5-min stage was the longest protocol $(28.93 \pm 3.50 \mathrm{~min})$; 1 - $\mathrm{min}$ was the shortest $(8.29 \pm 0.99 \mathrm{~min})$ and 3 -min protocol falls in between $(19.71 \pm 2.55 \mathrm{~min})$. According to Bayes Factor analyzes there is a chance of $99 \%$ to observe a difference among all stage lengths for time to exhaustion. Our data corroborate previous studies ${ }^{12}$. It has a practical application because a shorter test could save time. However, we need to know the best relationship between the shorter test and BGT objectivity. As we did not do the MLSS determination, we cannot respond to this question yet.

The maximum power obtained was different among stage length. The Bayes Factor analyses show that the 1min stage length has a probability of $96 \%$ and $99 \%$ to be higher than 3 and $5 \mathrm{~min}$, respectively. The 3-min stage length has $99 \%$ of the chance to be higher than a 5 -min one. The 5-min stage had the lowest maximum load, the 1min had the highest load, and a 3-min protocol falls in between both. The stage length is important to determine the boundary between severe and extreme domains. The peak load is used to prescribe the high-intensity interval training. Incremental tests with stages higher than $3 \mathrm{~min}$ seem to achieve lower $\mathrm{VO}_{2 \max }$ values ${ }^{12}$. Therefore, the optimal stage length applied in an incremental test is essential to improve the objectivity of submaximal and maximal parameters for exercise prescription.

As expected, the maximum heart rate was not different among stage length $\left(\mathrm{P}=0.24 ; \mathrm{\eta}_{\mathrm{p}}^{2}=0.11\right)$. The values obtained were $183 \pm 8.91(1 \mathrm{~min}) ; 187 \pm 11.15(3 \mathrm{~min})$; and $182 \pm 10.79(5 \mathrm{~min})$. The Bayes Factor showed a chance of $56 \%$ of similarity for maximum heart rate among stage length. The maximum heart rate has low applicability for training prescription and training adaptation assessment.

We chose recreational cyclists (people not engaged in systematic training and who do not compete) ${ }^{20}$ to have more homogeneity and because they were physical education students in our university the chance of subjects to withdraw from the study decreased. As Science is made to reach a generalization of conclusions, we believe that the sample characteristics do not limit our data. Therefore, the level of physical fitness seems not to be decisive in choosing the best length chosen to determine the BGT, and we can generalize the data obtained here.

A possible limitation of the present study was the lack of the MLSS determination from the subjects. However, our research group have already published data showing that MLSS can be estimated by BGT, using the protocol with 3 min per stage in an incremental exercise. The randomization was not counterbalanced, which could be another limitation. However, the study number of participants was adequate to reach the alfa level of $5 \%$ and the beta of $80 \%$. Another open gap is the intensity manipulation in an incremental test. We did not modulate this variable, and further work must take it into account. Nevertheless, it is likely that these limitations may not alter the conclusions obtained by this study.

\section{Conclusion}

We concluded that the stage length influences the BGT intensity determination. Although the BGT has a high intraclass correlation among evaluators, regardless of stage length, only one evaluator can decrease the objectivity of BGT. Therefore, we recommend the BGT as useful tool mainly due to its easy determination, good agreement among evaluators, non-expensive method and training-sensitivity. Nevertheless, two evaluators are needed at least; the evaluator must be cautious to choose the stage length; submaximal heart rate is affected by stage length, as well as the time to exhaustion and maximum load. 


\section{Acknowledgments}

The authors would like to thank the participants who contributed to the data collection in and the English revision made by Carmen Andrea Perez.

\section{References}

1. Simoes HG, Grubert Campbell CS, Kokubun E, Denadai BS, Baldissera V. Blood glucose responses in humans mirror lactate responses for individual anaerobic threshold and for lactate minimum in track tests. Eur J Appl Physiol Occup Physiol. 1999;80(1):34-40. PubMed PMID: 10367721. Epub 1999/06/15. eng.

2. Simões HG, Hiyane WC, Benford RE, Madrid B, Prada FA, Moreira SR, et al.. Lactate threshold prediction by blood glucose and rating of perceived exertion in people with type 2 diabetes. Percept Mot Skills. 2010;111(2):365.

3. Svedahl K, MacIntosh BR. Anaerobic threshold: the concept and methods of measurement. Canadian Journal of Applied Physiology. 2003;28(2):299-323.

4. Beneke R, Hutler M, Leithauser RM. Maximal lactatesteady-state independent of performance. Med Sci Sports Exerc. 2000;32(6):1135-9. PubMed PMID: 10862542. Epub 2000/06/22. eng.

5. Sotero RC, Pardono E, Landwehr R, Campbell CS, Simoes HG. Blood glucose minimum predicts maximal lactate steady state on running. Int J Sports Med. 2009;30(9):643-6. PubMed PMID: 19569005. Epub 2009/07/02. eng.

6. Motoyama YL, Pereira PEdA, Esteves GdJ, Duarte JMP, Carrara VCP, Rissato GM, et al.. Alternative methods for estimating maximum lactate steady state velocity in physically active young adults. Revista Brasileira de Cineantropometria \& Desempenho Humano. 2014;16(4):419-26.

7. Rosa TS, Simoes HG, Rogero MM, Moraes MR, Denadai BS, Arida RM, et al.. Severe Obesity Shifts Metabolic Thresholds but Does Not Attenuate Aerobic Training Adaptations in Zucker Rats. Front Physiol. 2016;7:122. PubMed PMID: 27148063. Pubmed Central PMCID: PMC4835489. Epub 2016/05/06. eng.

8. Rocha C, Canellas A, Monteiro D, Antoniazzi M, Azevedo $\mathrm{PH}$. Changes in individual glucose threshold during military training. Int J Sports Med. 2010;31(7):482-5. PubMed PMID: 20425684. Epub 2010/04/29. eng.

9. Junior PB, de Andrade VL, Campos EZ, Kalva-Filho CA, Zagatto AM, de Araujo GG, et al. Effect of Endurance Training on The Lactate and Glucose Minimum Intensities. J Sports Sci Med. 2018;17(1):117-23. PubMed PMID: 29535585. Pubmed Central PMCID: PMC5844198. Epub 2018/03/15. eng.

10. Bentley DJ, Newell J, Bishop D. Incremental exercise test design and analysis: implications for performance diagnostics in endurance athletes. Sports Med. 2007;37(7):57586. PubMed PMID: 17595153. Epub 2007/06/28. eng.
11. Faude O, Kindermann W, Meyer T. Lactate threshold concepts: how valid are they? Sports Med. 2009;39(6):469-90. PubMed PMID: 19453206. Epub 2009/05/21. eng.

12. Jamnick NA, Botella J, Pyne DB, Bishop DJ. Manipulating graded exercise test variables affects the validity of the lactate threshold and [Formula: see text]. PLoS One. 2018;13 (7):e0199794. PubMed PMID: 30059543. Pubmed Central PMCID: PMC6066218. Epub 2018/07/31. eng.

13. World Medical Association Declaration of Helsinki: ethical principles for medical research involving human subjects. J Am Coll Dent. 2014;81(3):14-8. PubMed PMID: 25951678. Epub 2015/05/09. eng.

14. WESTFALL PH, JOHNSON WO, UTTS JM. A Bayesian perspective on the Bonferroni adjustment. Biometrika. 1997;84(2):419-27.

15. Jeffreys H. The theory of probability: OUP Oxford; 1998.

16. Lee MD, Wagenmakers E-J. Bayesian cognitive modeling: A practical course: Cambridge university press; 2014.

17. Quintana DS, Williams DR. Bayesian alternatives for common null-hypothesis significance tests in psychiatry: a nontechnical guide using JASP. BMC Psychiatry. 2018;7,18 (1):178. PubMed PMID: 29879931. Pubmed Central PMCID: PMC5991426. Epub 2018/06/09. eng.

18. Rose AJ, Richter EA. Skeletal muscle glucose uptake during exercise: how is it regulated? Physiology (Bethesda). 2005;20:260-70. PubMed PMID: 16024514. Epub 2005/07/ 19. eng.

19. Meyer T, Lucia A, Earnest CP, Kindermann W. A conceptual framework for performance diagnosis and training prescription from submaximal gas exchange parameterstheory and application. Int J Sports Med. 2005;26 Suppl 1: S38-48. PubMed PMID: 15702455. Epub 2005/02/11. eng.

20. De Pauw K, Roelands B, Cheung SS, de Geus B, Rietjens G, Meeusen R. Guidelines to classify subject groups in sport-science research. Int J Sports Physiol Perform. 2013;8 (2):111-22. PubMed PMID: 23428482. Epub 2013/02/23. eng.

\section{Corresponding author}

Paulo Azevedo, Federal University of São Paulo, Ana costa, 95, $3^{\text {rd }}$ floor, Vila Mathias, Santos, SP, Brazil.

Email: paulo.azevedo@unifesp.br.

Manuscript received on November 8, 2018

Manuscript accepted on January 27, 2020

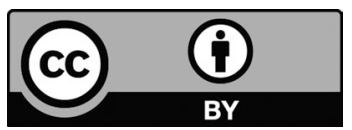

Motriz. The Journal of Physical Education. UNESP. Rio Claro, SP, Brazil - eISSN: 1980-6574 - under a license Creative Commons - Version 4.0 